Check for updates

Cite this: Phys. Chem. Chem. Phys., 2019, 21, 1089

Received 6th September 2018, Accepted 2nd December 2018

DOI: $10.1039 / c 8 c p 05665 j$

rsc.li/pccp

\title{
A systematic study of various 2D materials in the light of defect formation and oxidation $\dagger$
}

\author{
A. Dabral, (D)*ab A. K. A. Lu, (D) ${ }^{c}$ D. Chiappe, ${ }^{d}$ M. Houssa ${ }^{a b}$ and G. Pourtois ${ }^{\text {be }}$
}

\begin{abstract}
The thermodynamic aspects of various 2D materials are explored using Density Functional Theory (DFT). Various metal chalcogenides $\left(M X_{2}, M=\right.$ metal, chalcogen $\left.X=S, S e, T e\right)$ are investigated with respect to their interaction and stability under different ambient conditions met in the integration process of a transistor device. Their interaction with high- $\kappa$ dielectrics is also addressed, in order to assess their possible integration in Complementary Metal Oxide Semiconductor (CMOS) field effect transistors. 2D materials show promise for high performance nanoelectronic devices, but the presence of defects (vacancies, grain boundaries,...) can significantly impact their electronic properties. To assess the impact of defects, their enthalpies of formation and their signature levels in the density of states have been studied. We find, consistently with literature reports, that chalcogen vacancies are the most likely source of defects. It is shown that while pristine 2D materials are in general stable whenever set in contact with different ambient atmospheres, the presence of defective sites affects the electronic properties of the $2 \mathrm{D}$ materials to varying degrees. We observe that all the $2 \mathrm{D}$ materials studied in the present work show strong reactivity towards radical oxygen plasma treatments while reactivity towards other common gas phase chemical such as $\mathrm{O}_{2}$ and $\mathrm{H}_{2} \mathrm{O}$ and groups present at the high- $\kappa$ surface varies significantly between species. While energy band-gaps, effective masses and contact resistivities are key criteria in selection of $2 \mathrm{D}$ materials for scaled CMOS and tunneling based devices, the phase and ambient stabilities might also play a very important role in the development of reliable nanoelectronic applications.
\end{abstract}

\section{Introduction}

Silicon based devices have been at the forefront of device technologies for decades, allowing for continuous scaling in the micron and the sub-micron domains. As we move deeper into the nanometer regime, the usual solutions of voltage and channel scaling start breaking down. Phenomena such as short channel effects, leakage currents and quantum phenomena in the nanometer regime pose significant hindrance to further scaling and improvement of transport properties. ${ }^{1}$ Novel device architectures and materials have been proposed to solve these issues that plague CMOS technology. For instance, Tunnel Field Effect Transistors (TFET), ${ }^{2}$ Junction Field Effect Transistors (JFET), and Carbon Nanotube (CNT) ${ }^{3}$ Fin-FET ${ }^{4}$ and spin logic based devices $^{5}$ are some of the device technologies that are currently being considered as possible alternatives to classical CMOS.

\footnotetext{
${ }^{a}$ Ku Leuven, 3000 Leuven, Belgium

${ }^{b}$ IMEC, 3001 leuven, Belgium

${ }^{c}$ MathAM-OIL, AIST \& Tohuku University, Japan

${ }^{d}$ ASM, Helsinki, Finland

${ }^{e}$ Plasmant, University of Antwerp, Belgium

$\dagger$ Electronic supplementary information (ESI) available. See DOI: 10.1039/ c8cp05665j
}

The use of 2D materials as alternate channels has gathered significant interest over the last decade. ${ }^{6,7}$ They are predicted to show high performance in nanoelectronic devices. ${ }^{8}$ For example, graphene based devices showed promise due to their extremely high carrier mobilities. ${ }^{9}$ The discovery of graphene also opened up avenues for the investigation of various other 2D materials with exceptional properties. Perfect monolayers of $2 \mathrm{D}$ materials promise the absence of dangling bonds due to their self-limited nature and to the van der Waals (VdW) interactions existing between the layers. Further, the subnanometer thickness of the monolayers in 2D materials is ideal for their use as channel materials since they don't suffer from quantization effects induced along a third dimension. Consequently, the interest in $2 \mathrm{D}$ materials has driven the exploration and the identification of new candidates. At present, a wide variety of materials have been discovered or predicted to be stable via first principles techniques. ${ }^{10}$ This vast wealth in composition offers the premise to be able to engineer improved subthreshold slopes in electron devices, ideal scaling and low power consumption and opens the door for the development of novel applications in a multitude of domains ${ }^{11}$ provided that the materials meet the expectations in terms of electronic properties and stability. Graphene, for example, 'suffers' from 
a zero bandgap which renders it inapt for channel materials. Similarly, many other 2D materials may have metallic, semimetallic or semiconducting nature depending on the phase and stability which limit their use as device materials. Further, the currently grown 2D materials in current industrial tools deviate from the ideal perfect picture we have. The presence of intrinsic and extrinsic defects makes them sensitive to gas species present in their environment such as $\mathrm{H}_{2} \mathrm{O}, \mathrm{O}_{2}$, and $\mathrm{O}_{3}$. For example, in a water based $2 \mathrm{D}$ transfer process ${ }^{12,13}$ going from a grown substrate to a CMOS compatible one, the extent of the defect reactivity with $\mathrm{H}_{2} \mathrm{O}$ can be crucial in determining the quality of the devices and in the understanding of whether the material can be oxidized or not. Similarly, the presence of molecular oxygen in the environment may lead to the oxidation of 2D materials at different stages of the device integration steps (growth of the dielectrics, layer transfer $e t c$.). Interactions with the environment can hence lead to significant modulation of electronic properties such as their bandgap $\left(E_{\mathrm{g}}\right)$ and density of states (DOS) depending on the extent of their reactivity. The presence of detrimental effects induced by the environment may require the development of encapsulation approaches of the devices or some specialized processing and transfer techniques. A careful analysis of these aspects is hence needed in order to identify the most promising candidates capable of meeting all the requirements of future transistors.

In this work, we present a comprehensive analysis of the thermodynamics of $2 \mathrm{D}$ materials w.r.t. their reactivity in the presence of different environments typically met during the different steps in their integration in a transistor device. Using firstprinciples simulations to obtain qualitative guidelines for the material selection, we show that while pristine $2 \mathrm{D}$ materials are stable under most of the ambient conditions met in CMOS processing, defective ones have on the contrary strong reactivity. We find, consistently with literature ${ }^{14}$ reports, that chalcogen vacancies are the most likely source of defects. Even though we studied isolated defects, our qualitative findings can easily be generalized to the case of grain boundaries and ribbon edges as they represent possible sites of defects and reactions. Our results reveal that thermodynamic considerations in terms of stability and interaction with the surrounding medium of $2 \mathrm{D}$ materials can be used as additional criteria to identify the most suitable materials for $2 \mathrm{D}$ based nanoelectronics.

\section{Methodology}

In order to analyze the reactivity of $2 \mathrm{D}$ materials, we evaluated the enthalpy of reaction, $\Delta H$, of different oxidizing agents and oxygen containing sources like diatomic oxygen $\left(\mathrm{O}_{2}\right)$, radical $\mathrm{O}$, dielectric hydroxyl groups $(\mathrm{OH})$ and water $\left(\mathrm{H}_{2} \mathrm{O}\right)$. The thermodynamic driving forces of the reactions are approximated by the enthalpy of reaction of the system at $0 \mathrm{~K}$, hereby neglecting any entropic and pressure-volume contributions below.

$$
\Delta G(p, T)=\Delta H(p, T)-T \Delta S
$$

Since, typically, the energetic contribution $(\Delta H)$ strongly dominates the entropic term $(T \Delta S)$, the examination of $\Delta H$ provides qualitative insights into the spontaneous character of the reactions. For the sake of simplicity, we do not make any differentiation between $\Delta H, \Delta G$ and $\Delta E$. For example, the reaction depicting the formation of a chalcogen vacancy $\mathrm{X}_{\mathrm{V}}$ is given by

$$
\mathrm{MX}_{2} \rightarrow \mathrm{MX}_{2}\left(\mathrm{X}_{\mathrm{V}}\right)+\mathrm{X}(\uparrow \text { gas, } \downarrow \text { solid })
$$

and the corresponding formation energy (enthalpy) of $\mathrm{X}_{\mathrm{V}}, \Delta H_{\mathrm{XV}}$, is given by

$$
\Delta H_{\mathrm{XV}}=E_{\mathrm{MX}_{2}}\left(\mathrm{X}_{\mathrm{V}}\right)+\mu_{\mathrm{X}}-E_{\mathrm{MX}_{2}}
$$

where $E_{\mathrm{Mx}_{2}}\left(\mathrm{X}_{\mathrm{V}}\right)$ and $E_{\mathrm{Mx}_{2}}$ are the total energies of the $2 \mathrm{D}$ material with and without a vacancy and $\mu_{\mathrm{X}}$ is the chemical potential of the released element (X). $\mu$ can vary either from a solid or a gas source, depending on whether the atom removed to form the vacancy is set in a gaseous form or in a precipitate after the reaction.

We used the first-principle CP2K package ${ }^{15}$ combined with the Perdew-Burke-Ernzerhof (PBE) ${ }^{16}$ exchange-correlation functional and Goedecker-Teter-Hutter $(\mathrm{GTH})^{17}$ pseudo-potentials (PP) to model our systems. We used a $5 \times 5 \times 1$ repetition of the primitive unit cell of the $2 \mathrm{D}$ material (see Fig. 1) to model the reactions of the $2 \mathrm{D}$ materials, which ensures that the system can be integrated with one $k$ point (gamma), such that a good balance between the number of atoms used and the computational burden needed to simulate the stack can be obtained.

Further, it ensures that the system is large enough to prevent artificial interaction of the defects with its periodic image. High planewave cutoffs ( $>350 \mathrm{Ry}$ ) for atoms in $\mathrm{MX}_{2}$ were used as compared to the default 280 Ry to obtain better convergence. A defect/vacancy is created by removing one of the atoms as shown in Fig. 1b. The coordinates of the unit cell were extracted from ref. 18. van der Waals (vdW) corrections have been included for all systems through the use of the DFT-D3 Grimme formalism. ${ }^{19}$ The range of the vdW potential is set to be $10 \AA$. Individual atomic energies for different atoms were calculated using the self-consistent energy-force method (SCF) in CP2K with a double- $\varsigma$ polarization basis set. The atomic coordinates and the parameter of the unit cell describing the systems were made to be fully relaxed using a BFGS minimizer until convergence with a maximum atomic force criterion of $0.0002\left(\mathrm{bohr}^{-1}\right.$ hartree) was reached. a)
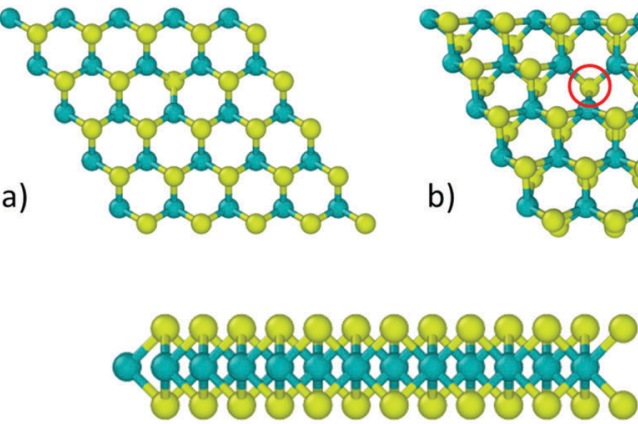

c)
Fig. 1 A $5 \times 5 \times 1 \mathrm{MoS}_{2}$ supercell (75 atoms). (a) Pristine $\mathrm{MoS}_{2}$ and (b) $\mathrm{MoS}_{2}$ with one sulfur vacancy circled in red. (c) A side view of a pristine $\mathrm{MoS}_{2}$ monolayer. 


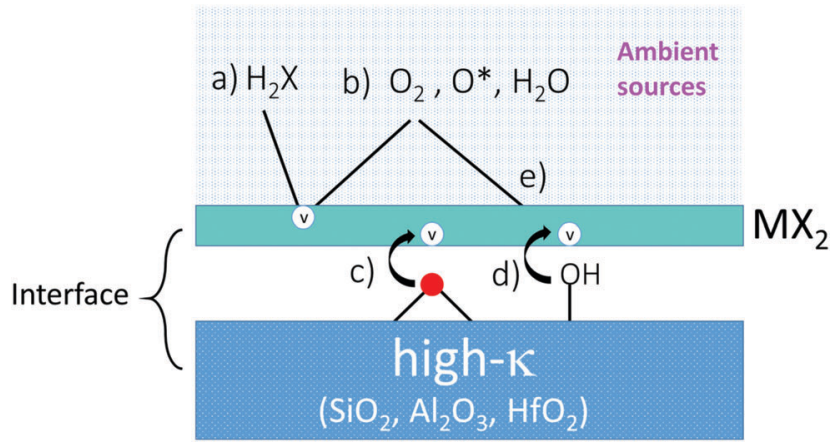

Fig. 2 Schematic illustration of the various scenarios depicting the reaction mechanisms of a $2 \mathrm{D}$ material $\left(\mathrm{MX}_{2}\right)$ with its environment. Circled $v$ represents a vacancy in a $(5 \times 5 \times 1)$ supercell, either surface or interfacial. (a) A reducing agent $\mathrm{H}_{2} \mathrm{X}$ source is acting on a vacancy. (b) Different oxidizing species present in the environment: the $\mathrm{O}_{2}$ molecule, oxygen plasma $\left(\mathrm{O}^{*}\right)$ and the water molecule. (c) A high- $\kappa$ surface atom interacting with an interface vacancy. (d) Interfacial oxidizing species like $-\mathrm{OH}$ in the form of high- $\kappa$ surface groups. (e) Pristine surface with no vacancy.

In order to emulate the impact of different chemical environments, the total energies from bulk and gas phases for both metal and chalcogen atoms were used to evaluate their chemical potentials. This allows establishing the impact of the evolution of formation energies, to mimic for instance the conditions of element sputtering in the gas phase and their precipitation.

In order to assess the impact of device processing and integration conditions on 2D materials under various conditions, we defined different scenarios (Fig. 2) depicting the interaction of 2D materials with some of the most common ambient species met in CMOS processing. Fig. 2 represents a schematic illustration of the possible interaction schemes with different oxidation sources of a $2 \mathrm{D}$ material $\left(\mathrm{MX}_{2}\right)$ vacancy.

In CMOS processing steps, different oxidizing sources can be used during either the transfer or the deposition of the dielectric. For instance: oxygen molecules, oxygen plasma, water molecules and/or reducing agents like $\mathrm{H}_{2} \mathrm{X}$ (chalcogen $\mathrm{X}=\mathrm{S}, \mathrm{Se}, \mathrm{Te}, \ldots$ ) can be introduced to reduce the defectivity of the $2 \mathrm{D}$ material. ${ }^{20}$ Further interactions at the 2D high- $\kappa$ dielectric interface can also occur in a transfer process, where the hydroxyl groups of the dielectric surface can oxidize a defective center in $\mathbf{M X}_{2}$.

The impact of defects and/or oxidation of the 2D materials on the electronic density of states (DOS) of 2D materials is also investigated. The DOS calculations are done with $\mathrm{CP} 2 \mathrm{~K}$ using the gamma point. A Gaussian spread $\sigma=0.1 \mathrm{eV}$ has been used for all materials. Note that a single $k$ point results in low resolution of the density of states. We can, however, get a qualitative idea of the presence of defect states in the gap and the modulation of the DOS due to the presence of various species.

\section{Results and discussion}

\subsection{Enthalpy of formation of vacancies}

Formation enthalpies of vacancies were evaluated for about 40 different materials (sulfides, selenides and tellurides) using eqn (3). Fig. 3 and 4 show the $\Delta H_{\mathrm{V}}$ computed for the formation

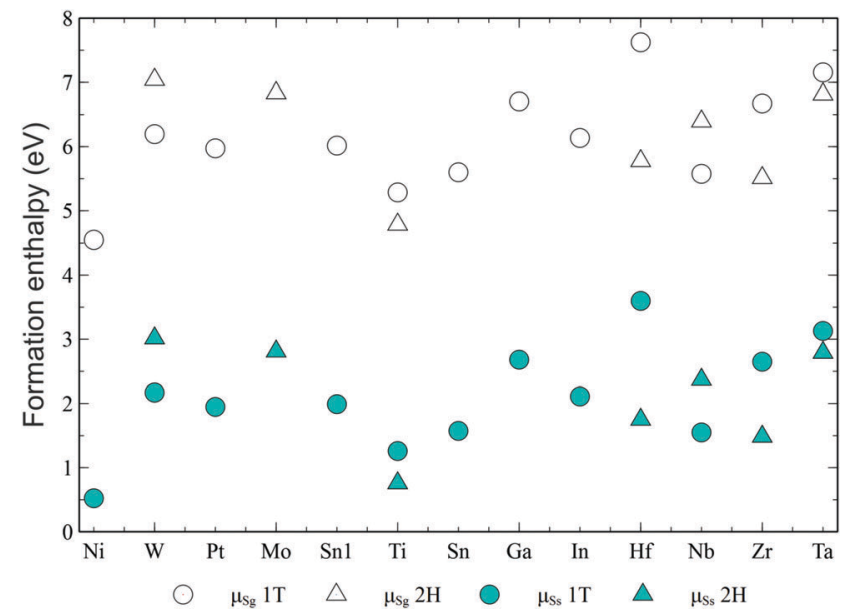

Fig. $3 \Delta H_{\mathrm{Sv}}$ (formation energy of sulfur vacancies) assuming the release of sulfur in the gas (open symbols) and in a solid phase (closed symbols) is shown on the vertical axis. The horizontal axis shows the coordinating cation used in $M X_{2}$. Whenever identified, the impact of different phases of the $2 \mathrm{D}$ material $(1 \mathrm{~T} / 2 \mathrm{H})$ is listed for a particular material.

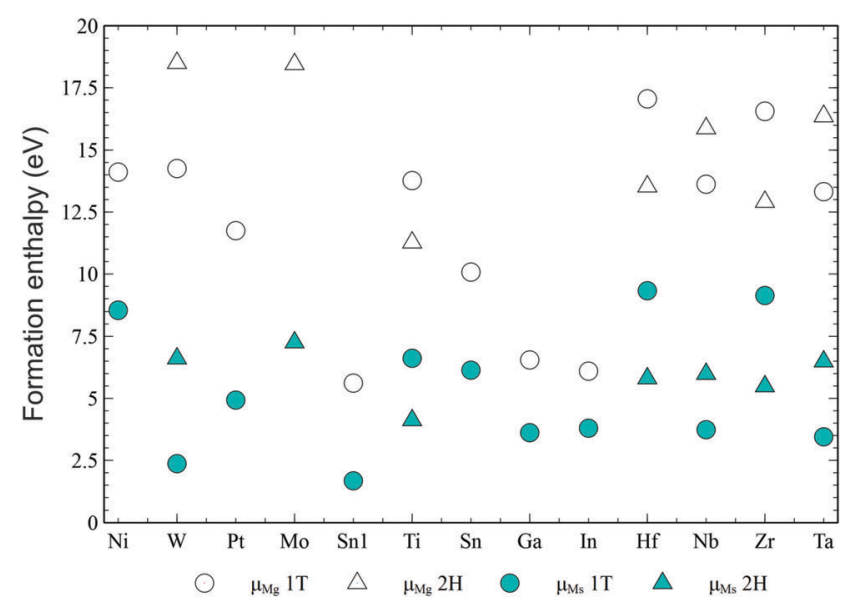

Fig. $4 \Delta H_{M v}$ (formation energy of metal vacancies) assuming the release of metal in the gas (open symbols) and in a solid phase (closed symbols) is shown on the vertical axis. The horizontal axis shows the coordinating cation used in $\mathrm{MX}_{2}$. Whenever identified, the impact of different phases of the $2 \mathrm{D}$ material is listed for a particular material. Circles and triangles correspond to the $1 \mathrm{~T}$ and $2 \mathrm{H}$ phases of $\mathrm{MX}$.

of sulfur and metal vacancies in various $2 \mathrm{D}$ materials, in different phases. The enthalpies were calculated using the chemical potential of sulfur extracted from an isolated atom, $\mu_{\mathrm{sg}}$, and from bulk sulfur, $\mu_{\mathrm{Ss}}$, to account for ambient conditions. They were compared below with appropriate references wherever possible. The formation enthalpies can be separated roughly in two sections based on their amplitude i.e. above and below $\sim 3.5 \mathrm{eV}$, as $\Delta H_{\mathrm{Sv}}$ varies significantly for $\mu_{\mathrm{Sg}}$ and $\mu_{\mathrm{Ss}}$. This suggests that the latter is energetically more favorable to occur. Note that for $\mathrm{MoS}_{2}$, we only report two values corresponding to the $2 \mathrm{H}$ phase, which is the semiconducting and more stable phase.

$\Delta H_{\mathrm{Sv}}$ for $\mu_{\mathrm{Ss}}$ is found to be $2.81 \mathrm{eV}$ in $\mathrm{MoS}_{2}-2 \mathrm{H}$, which lies within the range of values reported previously (between $2.6 \mathrm{eV}$ and $3.3 \mathrm{eV}$ in ref. 21-25). $\Delta H_{\mathrm{SV}}$ for sulfur vacancies in $\mathrm{MoS}_{2}-2 \mathrm{H}$ 
from $\mu_{\mathrm{Sg}}$ is $6.83 \mathrm{eV}$, which is slightly higher than calculated in earlier work i.e. $6.27 \mathrm{eV}^{26}$ and $4.44 \mathrm{eV} .^{27}$ The formation energy for a sulfur vacancy from $\mu_{\mathrm{Ss}}$ in the $\mathrm{HfS}_{2}-1 \mathrm{~T}$ stable phase is $3.49 \mathrm{eV}$ which is lower than the $4.40 \mathrm{eV}$ reported in ref. 28. For the less stable $\mathrm{HfS}_{2}-2 \mathrm{H}$ phase, both the gas and the solid phase sulfur lead to lower formation energies. For the $\mathrm{WS}_{2}-2 \mathrm{H}$ phase, $\Delta H_{\mathrm{Sv}}$ from $\mu_{\mathrm{Ss}}$ is $3.0 \mathrm{eV}$, which is close to $2.75 \mathrm{eV}$ mentioned in ref. 21. For GaS, $\Delta H_{\mathrm{Sv}}$ is $2.68 \mathrm{eV}$, which is higher than the $2.06 \mathrm{eV}$ reported in ref. 29. For $\mathrm{SnS}_{2}(\mathrm{Sn})$ there is only one stable phase. $\Delta H_{\mathrm{Sv}}$ is $1.57 \mathrm{eV}$ for $\mu_{\mathrm{Ss}}$ and $5.6 \mathrm{eV}$ for $\mu_{\mathrm{Sg}}$. The value for the gas phase is close to $5.05 \mathrm{eV}$ computed in ref. 30 and the ones for $\operatorname{SnS}(\mathrm{Sn} 1)$ are found to be higher than $\mathrm{SnS}_{2} . \Delta H_{\mathrm{Sv}}$ for $\mathrm{ZrS}_{2}-2 \mathrm{H}$ is given for both $1 \mathrm{~T}$ and $2 \mathrm{H}$ phases. The value is close to the one reported in ref. 30 for $\mu_{\text {sg. }}$. For the $\mathrm{WS}_{2}-2 \mathrm{H}$ stable phase, the formation energy is $3.0 \mathrm{eV}$ for $\mu_{\mathrm{Ss}}$, which is close to $2.76 \mathrm{eV}$ in ref. 21. For $\mathrm{WS}_{2}-2 \mathrm{H}, \mathrm{PtS}_{2}-1 \mathrm{~T}, \mathrm{TaS}_{2}-2 \mathrm{H}$ and $\mathrm{TiS}_{2}$, the vacancy formation energies from $\mu_{\mathrm{Sg}}$ are found to be in close agreement with the previously reported values in ref. 31 . In general, the formation energy of sulfur vacancies calculated from $\mu_{\mathrm{Sg}}$ is larger than the one calculated from $\mu_{\mathrm{Ss}}$, consistent with previously reported values.

Fig. 4 depicts the distribution of formation enthalpies of metal vacancies, $\Delta H_{\mathrm{Mv}}$, for the same metal chalcogenides as in Fig. 3. The difference in formation energies of metal vacancies, as compared to the chalcogen ones, is consistent with experimental observations, ${ }^{31}$ indicating their spontaneous formation to be very unlikely. Fig. 4 roughly separates the formation energies calculated from the chemical potentials of the isolated metal atoms, $\mu_{\mathrm{Mg}}$, and bulk metals, $\mu_{\mathrm{Ms}} . \Delta H_{\mathrm{Mv}}$ spans over a wide range. $\mathrm{MoS}_{2}-2 \mathrm{H}$ has a $\Delta H_{\mathrm{Mv}}$ of $7.24 \mathrm{eV}$, which is in agreement with previous work..$^{21,22,24}$ For $\mathrm{HfS}_{2}-1 \mathrm{~T} \Delta H_{\mathrm{Mv}}$ for $\mu_{\mathrm{Ms}}$ is $9.3 \mathrm{eV}$ which is also close to the reported value in ref. 28 and the one for GaS is $3.61 \mathrm{eV}$, which is in accord with $3.81 \mathrm{eV}$ reported in ref. 29. Similarly for $\mathrm{SnS}_{2}(\mathrm{Sn}), \Delta H_{\mathrm{Mv}}$ using $\mu_{\mathrm{Mg}}$ is found to be $10.0 \mathrm{eV}$, which is close to the $9.12 \mathrm{eV}$ reported in ref. 30. The values for $\mathrm{SnS}(\mathrm{Sn} 1)$ are lower than $\mathrm{SnS}_{2}$. It can also be seen that $\mathrm{SnS}_{2}$ is more prone to the formation of chalcogen vacancies, while $\mathrm{SnS}$ is more prone to $\mathrm{Sn}$ ones. $\Delta H_{\mathrm{Mv}}$ for $\mathrm{ZrS}_{2}-2 \mathrm{H}$ for $\mu_{\mathrm{Mg}}$ is $12.9 \mathrm{eV}$ which is slightly less than the one reported in ref. 30. For $\mathrm{WS}_{2}-2 \mathrm{H}, \Delta H_{\mathrm{Mv}}$ for $\mu_{\mathrm{Mg}}(6.56 \mathrm{eV})$ is found to be in agreement with ref. 21 .

In general, formation energies for metal vacancies are found to be higher than those of chalcogen vacancies, except for SnS(Sn1). This suggests that the natural abundancy of Sn vacancies is higher than S (Fig. 3 and 4 ) in this latter material. Note that only the important (or existing) phases for $\mathrm{SnS}, \mathrm{SnS}_{2}$, GaS, GaSe, InS, and InSe were explored in this work. The presence of atomic vacancies may induce states either close to the conduction or to the valence bands. Consistent with experimental observations, metal vacancies induce states close to the valence band, while chalcogen vacancies lead to states close to the conduction band. Interestingly, some 2D materials do not show gap states and seem to be defect tolerant (see $\left.\mathrm{HfS}_{2}-1 \mathrm{~T}\right)$. The impact of defects and oxidation on electronic properties is discussed in Section 4. For the sake of completeness, the distribution of the chalcogen and the metal vacancy formation energies for selenides and tellurides are provided in the ESI. $\dagger$ The formation energy trends for selenides and tellurides remain similar, with chalcogen vacancies being more favorable than metal vacancies. Tellurides have the lowest formation energies, while selenides lie between sulfides and tellurides, in general. The formation energy, as expected, varies from phase to phase $(1 \mathrm{~T} / 2 \mathrm{H})$ if present for a particular $2 \mathrm{D}$ material.

\subsection{Interaction of pristine $2 \mathrm{D}$ materials with the environment}

Various species like $\mathrm{O}_{2}, \mathrm{O}^{*}$ (plasma), $\mathrm{H}_{2} \mathrm{O}$ and high- $\kappa$ surface groups are typically present during the integration steps of $2 \mathrm{D}$ materials in nanoelectronic devices, either during their processing or electrical characterization steps. Therefore, it is important to ensure that the grown $2 \mathrm{D}$ materials are robust enough to maintain their properties, independent of the environment they are exposed to. A general overview of the interaction of pristine 2D materials in the presence of these oxidizing sources is shown in Fig. 5.

The impact of the environment and the surface chalcogen atoms is estimated by varying the chemical potential of the $\mathrm{O}$ atom, $\mu_{\mathrm{O}}$, extracted from different molecules $\left(\mathrm{O}^{*}, \mathrm{O}_{2}\right.$, and $\left.\mathrm{H}_{2} \mathrm{O}\right)$ :

$$
\begin{gathered}
\mathrm{MX}_{2}+\mathrm{H}_{2} \mathrm{O} \rightarrow \mathrm{MX}_{2}(\mathrm{O})+\mathrm{H}_{2} \\
\mathrm{MX}_{2}+\mathrm{O}_{2} \rightarrow \mathrm{MX}_{2}(\mathrm{O})+1 / 2 \mathrm{O}_{2} \\
\mathrm{MX}_{2}+\mathrm{O}^{*} \rightarrow \mathrm{MX}_{2}(\mathrm{O})
\end{gathered}
$$

Interestingly, all the $\mathrm{MX}_{2}$ candidates studied show the same trend: the exposure to radical oxygen (as typically used during the atomic layer growth of a dielectric on a $\mathrm{MX}_{2}$ channel) leads to strong exothermic chemisorption (oxidation) of all 2D materials, irrespective of the nature of the metal or the chalcogenide considered.

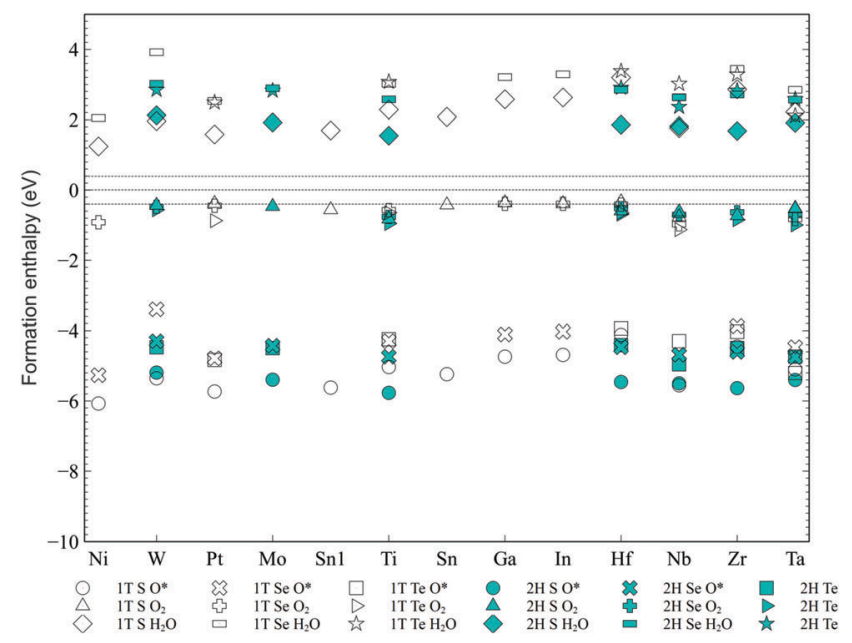

Fig. 5 Interaction of various species (labeled with different shapes) with pristine $2 \mathrm{D}$ materials. The metal cation is listed along the horizontal axis and $\Delta H$ of interaction is given on the vertical axis for different species. For example, $\mathrm{O}^{*} \mathrm{~S}$ (open circles) represents interaction of $\mathrm{O}$ (plasma) with 1T sulfides. The dotted horizontal lines show an approximate boundary around $0 \mathrm{eV}$ between physisorption (small negative/positive energies), chemisorption (large negative energies) and no interaction (high positive energies). $\mathrm{O}$ is highly oxidizing and $\mathrm{O}_{2}$ interacts in the physisorption range while $\mathrm{H}_{2} \mathrm{O}$ has no interaction with the pristine $2 \mathrm{D}$ materials. 
The interaction with a milder oxidizing source such as $\mathrm{O}_{2}$ leads to milder reactions, with endo or exothermic reactions, depending on the system considered. $\mathrm{H}_{2} \mathrm{O}$ has positive enthalpies of reaction, and does not spontaneously interact with pristine 2D materials, except for some unstable phases. In the specific case of $\mathrm{MoS}_{2}-2 \mathrm{H}$, the adsorption energy of the $\mathrm{O}_{2}$ molecule (corresponding to the closed triangle in Fig. 5) is found to be exothermic $(\Delta H=-1.2 \mathrm{eV})$, which is in close agreement with the value reported $(-1.12 \mathrm{eV})$ in ref. 32. Without surprises, the less stable $1 \mathrm{~T}$ metallic phase reacts spontaneously with $\mathrm{O}_{2}(-7.7 \mathrm{eV}$, open triangle). Similar trends are observed for other 2D materials. The above analysis shows that most pristine $2 \mathrm{D}$ materials should be stable in air and water.

Interestingly, a closer look at the energies of interaction (eqn (7)) of an $\mathrm{O}_{2}$ molecule with the chalcogen layer $\mathrm{S}$ of $\mathrm{MX}_{2}$ (Fig. 6) reveals a large dependence of the inter-atomic distance between the 2D materials and the closest oxygen atom with respect to the nature of the metal and of the chalcogenide present.

$$
\mathrm{MX}_{2}+\mathrm{O}_{2} \rightarrow \mathrm{MX}_{2}\left(\mathrm{O}_{2}\right)
$$

This is accompanied by a strong modulation of the interaction energy between the two systems, which shows a qualitative linear evolution with the separating distance. In general, we observe that higher interaction energies correspond to shorter interatomic distances and vice versa. In the case of the $2 \mathrm{H}$ phase of $\mathrm{MoS}_{2}$, the interaction energy is $-0.47 \mathrm{eV}$ and the closest interatomic distance with oxygen is $3.25 \AA$. This suggests that $\mathrm{O}_{2}$ is weakly interacting (physisorption) with $\mathrm{MoS}_{2}$ in its pristine form. The reduction potential of different metals forming a $2 \mathrm{D}$ material might also have an impact on the interaction energy. In general, the interaction with $\mathrm{O}_{2}$ is phase and system dependent and the boundaries between a physisorption and a chemisorption process are unclear (while following an expected trend w.r.t. bond lengths). Metallic phases, such as those of $\mathrm{TiS}_{2}$ and $\mathrm{TaS}_{2}$, in general, have stronger interaction with $\mathrm{O}_{2}$, which

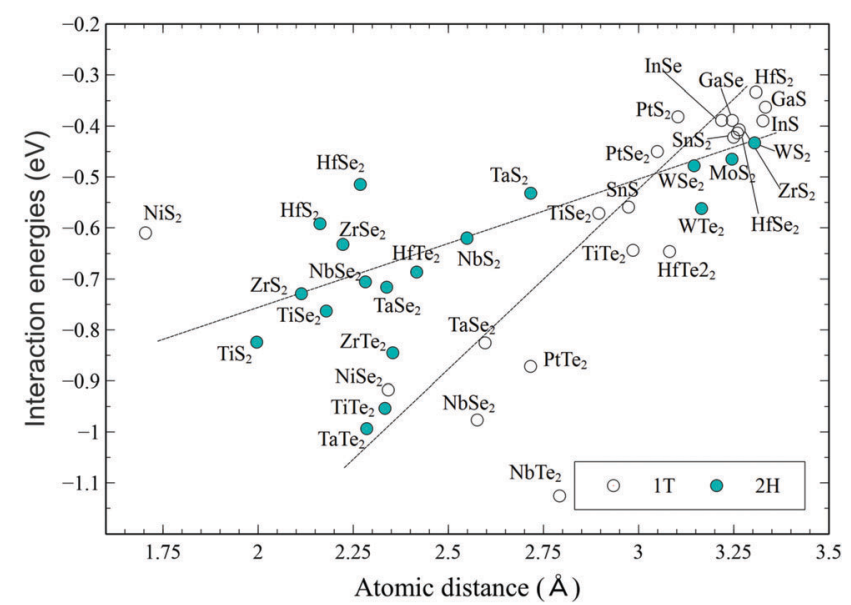

Fig. 6 The distribution of the atomic distance $(\AA \AA)$ vs. the resulting interaction energy of the $\mathrm{O}$ atom (in $\mathrm{O}_{2}$ ) lying nearest to the chalcogen atom on the surface of the $2 \mathrm{D}$ materials. Two guiding lines show the trend for $1 \mathrm{~T}$ and $2 \mathrm{H}$ phases. suggests that they are keener to undergo an oxidation process. ${ }^{33}$ Tellurides and selenides are found to be more reactive as compared to sulfides.

\subsection{Interaction of defective $2 \mathrm{D}$ materials with the environment}

Since the formation of chalcogen vacancies is the most likely source of defects in the $\mathrm{MX}_{2}$ materials, these are naturally present in the material. We therefore focus in this section on the oxidation of a single vacancy in contact with different ambient gas species. The reactivity of sulfide, selenide and telluride based $\mathrm{MX}_{2}$ is explored w.r.t. their interaction with $\mathrm{O}^{*}, \mathrm{H}_{2} \mathrm{O}$ and hydroxyl surface groups, as typically present in dielectric materials. While pristine $2 \mathrm{D}$ materials are mostly inert with respect to a non-plasma environment, the reactivity of defective 2D materials depends strongly on the type of material and on the oxidation source. As an illustration, Fig. 7 shows a comparison of the oxidation enthalpies of sulfides with $\mathrm{O}^{*}, \mathrm{H}_{2} \mathrm{O}$ and $\mathrm{SiO}_{2}$ surface hydroxyl groups $(-\mathrm{OH})$, corresponding to eqn (8)-(10). In the case of hydroxyl groups, the oxidation is by the $\mathrm{O}$ atom coming from the $-\mathrm{OH}$ surface group leaving behind $\mathrm{H}$ on the $\mathrm{SiO}_{2}$ surface.

$$
\begin{gathered}
\mathrm{MX}_{2}(\mathrm{~V})+\mathrm{O}^{*} \rightarrow \mathrm{MX}_{2}(\mathrm{O}) \\
\mathrm{MX}_{2}(\mathrm{~V})+\mathrm{H}_{2} \mathrm{O} \rightarrow \mathrm{MX}_{2}(\mathrm{O})+\mathrm{H}_{2} \\
\mathrm{MX}_{2}(\mathrm{~V})+\mathrm{SiO}_{2}-\mathrm{OH} \rightarrow \mathrm{MX}_{2}(\mathrm{O})+\mathrm{SiO}_{2}-\mathrm{H}
\end{gathered}
$$

The reaction enthalpies of defective $2 \mathrm{D}$ materials with $\mathrm{O}^{*}$ are all exo-energetic and summarized in Fig. 8. Interestingly, the enthalpies are more exo-energetic than those resulting from the interaction of $\mathrm{O}^{*}$ with pristine $2 \mathrm{D}$ materials. The differences in energies depend on the composition of the material and also the phase involved.

For example, $\mathrm{Ni}$ and $\mathrm{Ti}$ based $\mathrm{MX}_{2}$ have relatively lower reaction energies with $\mathrm{O}^{*}$ in both their pristine and defective forms,

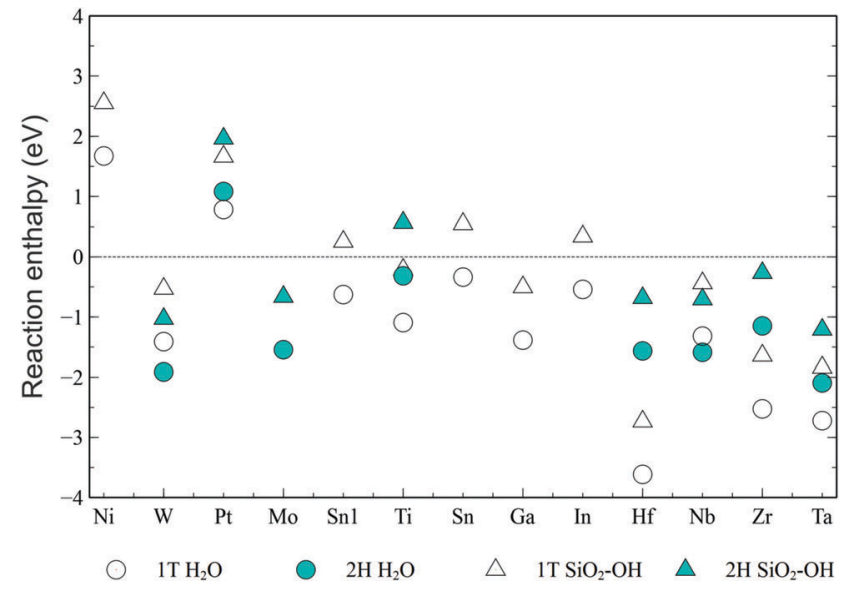

Fig. 7 Distribution of the oxidation enthalpies with $\mathrm{H}_{2} \mathrm{O}$ and $\mathrm{SiO}_{2}$ surface groups $(-\mathrm{OH})$ of a single chalcogen defect in sulfides. The interaction of $\mathrm{H}_{2} \mathrm{O}$ clearly has a larger impact, showing that defective 2D materials are less prone to react with surface groups. Open symbols and closed symbols correspond to $1 \mathrm{~T}$ and $2 \mathrm{H}$ phases respectively. 


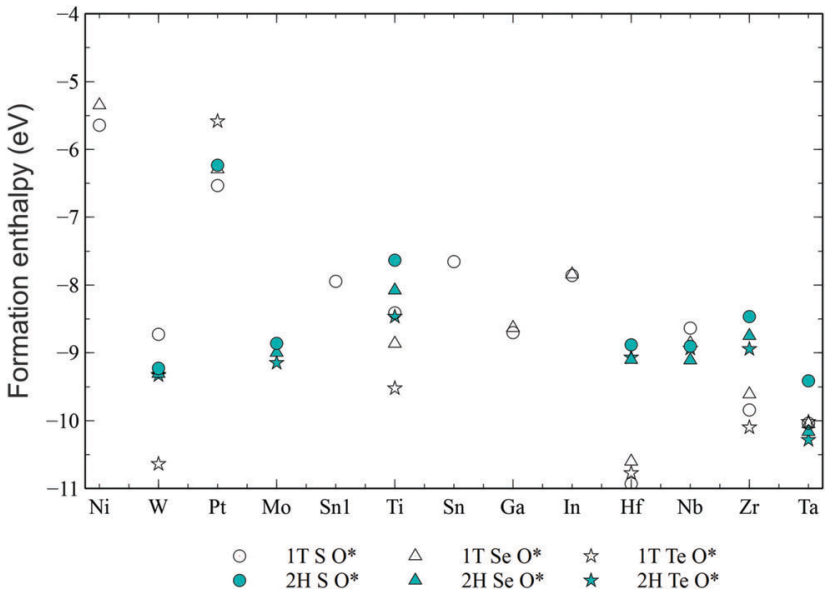

Fig. 8 Distribution of the oxidation enthalpies with $O^{*}$ (plasma) of a single chalcogen defect in different $2 \mathrm{D}$ materials. As expected, plasma oxidizes all 2D materials with defects with high negative enthalpies. Open symbols and closed symbols correspond to $1 \mathrm{~T}$ and $2 \mathrm{H}$ phases respectively.

while $\mathrm{MoS}_{2}$ behaves similarly to other materials in this regard. The reaction enthalpies of defective $2 \mathrm{D}$ materials with $\mathrm{H}_{2} \mathrm{O}$ (see Fig. 7) are in general less exo-energetic than those with the surface hydroxyl groups, showing that surface hydroxyl groups are less prone to react with defective $\mathrm{MX}_{2}$ materials than $\mathrm{H}_{2} \mathrm{O}$. They are nonetheless an important source of spontaneous oxidation. While the driving force for the oxidation by -OH groups is less pronounced, the reactivity for either $\mathrm{H}_{2} \mathrm{O}$ or $-\mathrm{OH}$ depends significantly on the phase for a fixed cation (Fig. 7).

The reaction enthalpies of defective $2 \mathrm{D}$ selenides and tellurides are given in Fig. 9 and 10 respectively; a similar trend can be seen in both cases. For instance, the reaction enthalpies for the $\mathrm{MoX}_{2}-2 \mathrm{H}$ stable phase with $\mathrm{H}_{2} \mathrm{O}$ are $-1.55 \mathrm{eV},-1.68 \mathrm{eV}$ and $-1.83 \mathrm{eV}$ when changing the chalcogenide from $\mathrm{S}$ to Se and Te respectively. This again shows that sulfur based $\mathbf{M X}_{2}$ are in general more stable than selenide and telluride ones.

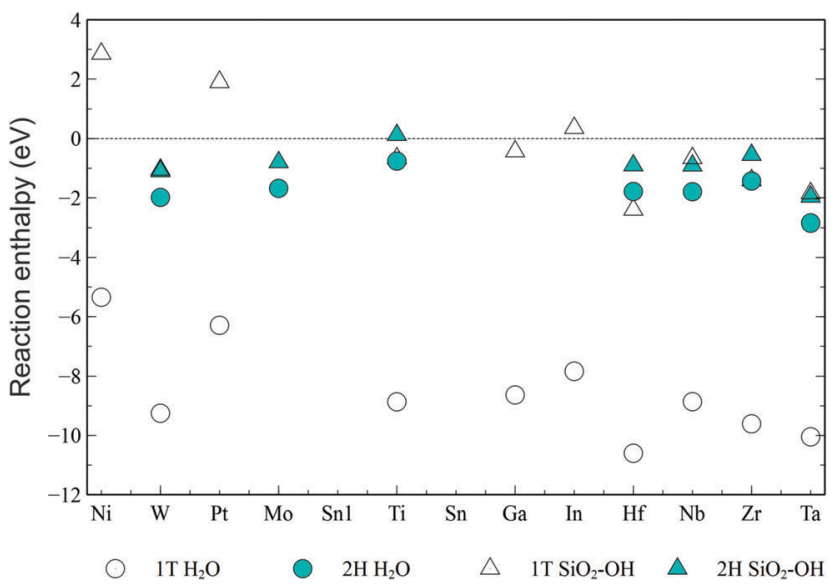

Fig. 9 Distribution of the oxidation enthalpies with $\mathrm{H}_{2} \mathrm{O}$ and $\mathrm{SiO}_{2}$ surface groups $(-\mathrm{OH})$ of a single chalcogen defect in selenides. The interaction of $\mathrm{H}_{2} \mathrm{O}$ (empty shapes) clearly has a larger impact showing that defective 2D materials are less prone to react with surface groups. Open and closed symbols correspond to $1 \mathrm{~T}$ and $2 \mathrm{H}$ phases respectively.

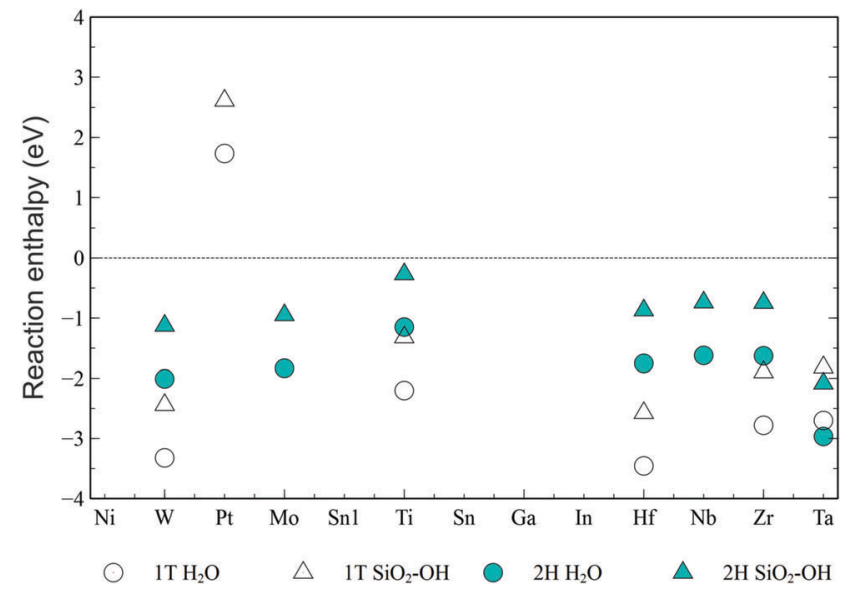

Fig. 10 Distribution of the oxidation enthalpies with $\mathrm{H}_{2} \mathrm{O}$ and $\mathrm{SiO}_{2}$ surface groups $(-\mathrm{OH})$ of a single chalcogen defect in tellurides. The interaction of $\mathrm{H}_{2} \mathrm{O}$ (empty circles) has a larger impact showing that defective 2D materials are less prone to react with surface groups. Open and closed symbols correspond to $1 \mathrm{~T}$ and $2 \mathrm{H}$ phases respectively.

The corresponding reaction enthalpies with hydroxyl groups present at the $\mathrm{SiO}_{2}$ surface are $-0.66 \mathrm{eV},-0.8 \mathrm{eV}$ and $-0.95 \mathrm{eV}$ respectively, which also reflect the same trend. The reaction enthalpies of defective $2 \mathrm{D}$ chalcogenides with $\mathrm{Al}_{2} \mathrm{O}_{3}-\mathrm{OH}$ and $\mathrm{HfO}_{2}-\mathrm{OH}$ (hydroxyl surface groups) are also found to be higher (more positive) as compared to $\mathrm{SiO}_{2}-\mathrm{OH}$ groups. However, the difference in reactivities is very small as shown for the case of sulfides (see Fig. S6, ESI $\dagger$ ).

\subsection{Impact of ambient interactions on electronic properties}

We consider now the electronic properties of $2 \mathrm{D}$ materials, and more specifically the impact of defects on their electronic structure. It has been shown that the vacancies in 2D materials can be utilized as dopants (n/p). ${ }^{34,35}$ For example, the presence of a chalcogen defect in the $\mathrm{MoS}_{2}-2 \mathrm{H}$ phase leads to states close to the conduction band while an Mo vacancy causes states closer to the valence band. While a chalcogen vacancy can be healed on oxidation (see the last section), the presence of defects can play an important role in controlling the doping in 2D materials. The partial electronic density of states is calculated via the projection onto atomic species, and the contribution of individual atoms is resolved. Alignment of the DOS is done w.r.t. hydrogen levels instead of the Fermi level to make a fair comparison using the methodology developed by Van De Walle. ${ }^{36}$ DOS of only the semiconducting phases of interest are shown. The highest occupied molecular orbital (HOMO level) is indicated with a vertical dashed line in each DOS plot.

Since we observe for all the systems studied the same qualitative trend, we will focus the discussion on the case of $\mathrm{MoS}_{2}-2 \mathrm{H}$. Sulfur vacancies, being more prominent, are discussed with respect to oxidation. The DOS for Mo vacancies in $\mathrm{MX}_{2}$ is provided for reference. The DOS of the pristine and defective (one chalcogen vacancy) phase is shown in Fig. 11a and $\mathrm{b}$ respectively.

The valence and conduction band states consist of both Mo and $\mathrm{S}$ contributions, and the signature of a clear defect state is 


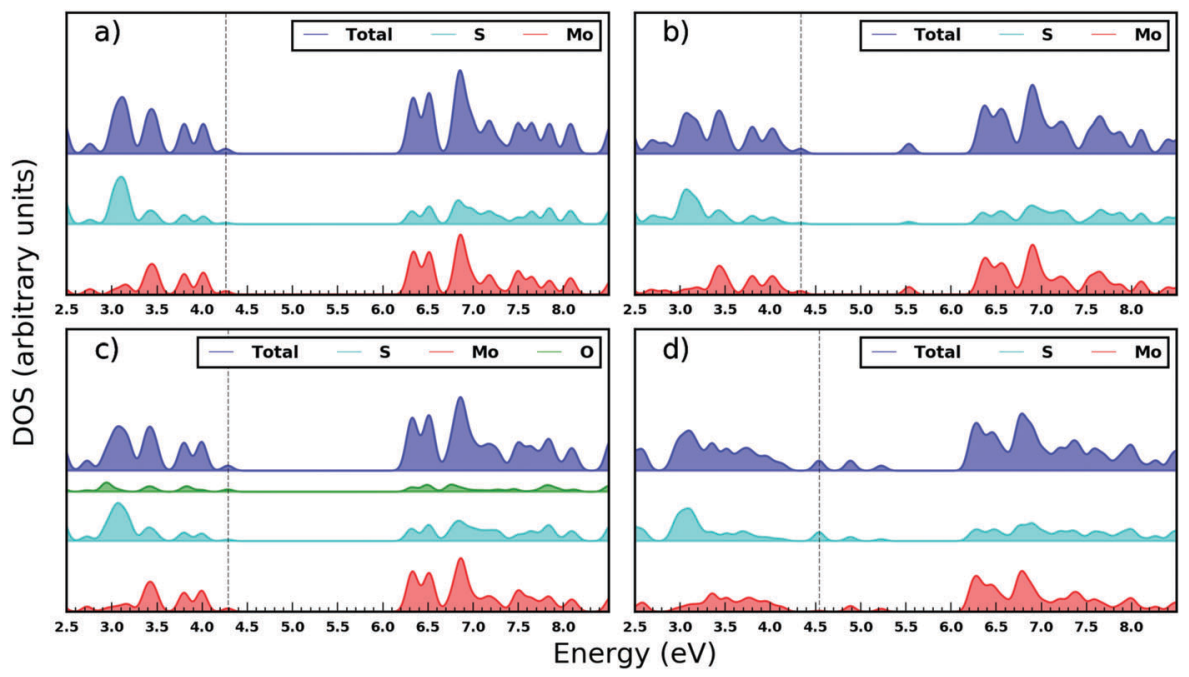

Fig. 11 (a) Shows the DOS for pristine $\mathrm{MoS}_{2}-2 \mathrm{H}$ with individual contributions. (b) Shows the DOS for MoS $2-2 \mathrm{H}$ with one sulfur vacancy. (c) Shows the DOS with the sulfur vacancy replaced by an oxygen atom. (d) Shows the impact of an Mo vacancy on the DOS. The vertical dashed line indicates the HOMO level.

observed in the forbidden gap at around $0.77 \mathrm{eV}$ below the $\mathrm{CB}$, due to a sulfur vacancy. This value is close to $0.6 \mathrm{eV}$ reported in ref. 37 , where a similar $5 \times 5 \times 1$ supercell has been used. The sulfur vacancy also introduces a shallow state close to the VB $(\sim 0.04 \mathrm{eV}$ above the VB) as noted in ref. 37 . The gap states are, as can be seen, made up of Mo and S orbitals with the major contribution coming from Mo. The three prominent states for the molybdenum vacancy, shown in Fig. 11d, are also comparable and extend up to the midgap region from the top of the valence band, as noted in ref. 37. We also see a modulation in the VB and the CB edges. The introduction of an oxygen atom replacing the sulfur vacancy site (oxidation of the vacancy) removes the gap state. The oxygen and sulfur atoms being iso-valent, oxygen saturates the dangling bonds of molybdenum at the sulfur vacancy site, as also shown in Fig. 11b. A similar observation was reported in ref. 38 . Note that since there is only one oxygen atom, the contribution of oxygen has been increased $20 \times$ to make it visible in the partial density of states. The contribution of the oxygen $\mathrm{p}$ orbitals is spread throughout the $\mathrm{CB}$ and VB of $\mathrm{MoS}_{2}$. There is a slight modification of the valence band close to the edge, due to the presence of oxygen states as can be seen from Fig. 11a and c.

A similar analysis is performed for $\mathrm{HfS}_{2}-1 \mathrm{~T}$ (stable phase) in Fig. 12. Fig. 12a shows the DOS for pristine $\mathrm{HfS}_{2}$. Unlike $\mathrm{MoS}_{2}$, the conduction band and valence band are mostly composed of the contribution of metal, Hf, and chalcogen, S, states respectively. Fig. 13b shows $\mathrm{HfS}_{2}-1 \mathrm{~T}$ with one chalcogen vacancy. It can be seen that a chalcogen vacancy does not introduce

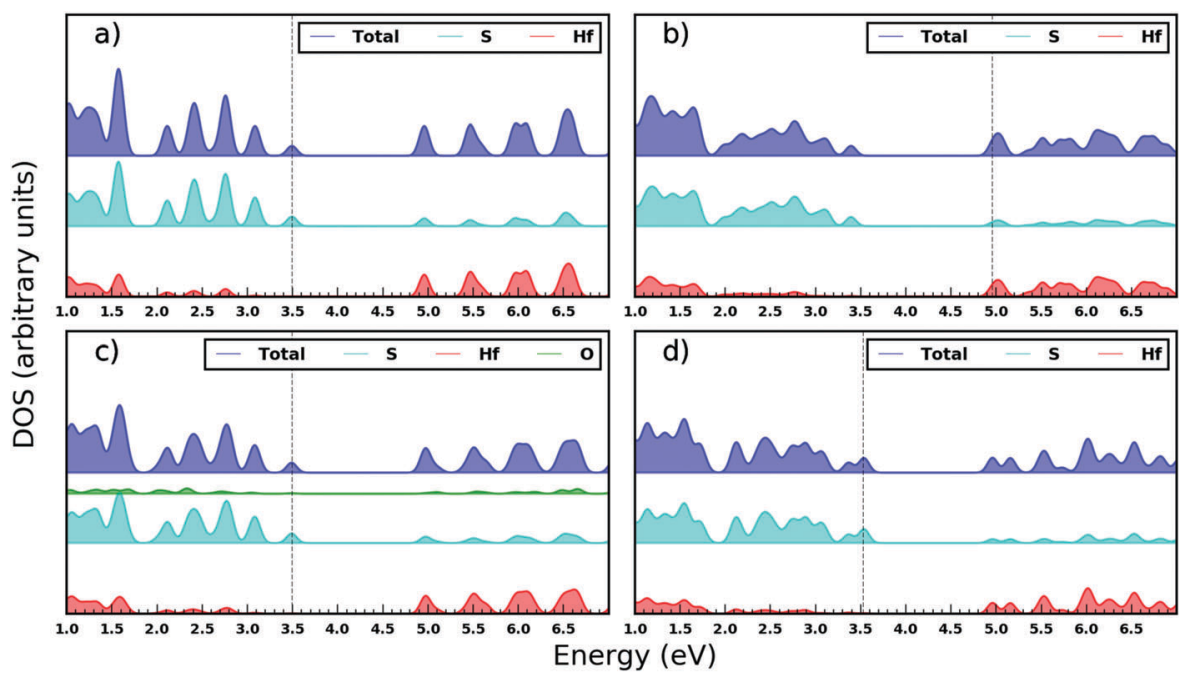

Fig. 12 (a) Shows the DOS for pristine $\mathrm{HfS}_{2}-1 \mathrm{~T}$ with individual contributions. (b) Shows the DOS for $\mathrm{HfS}_{2}-1 \mathrm{~T}$ with one sulfur vacancy. (c) Shows the DOS with the sulfur vacancy replaced by an oxygen atom. The contribution of oxygen states is relatively higher close to the valence band edge. (d) Shows the impact of an $\mathrm{Hf}$ vacancy on the DOS. The vertical dashed line indicates the HOMO level. 


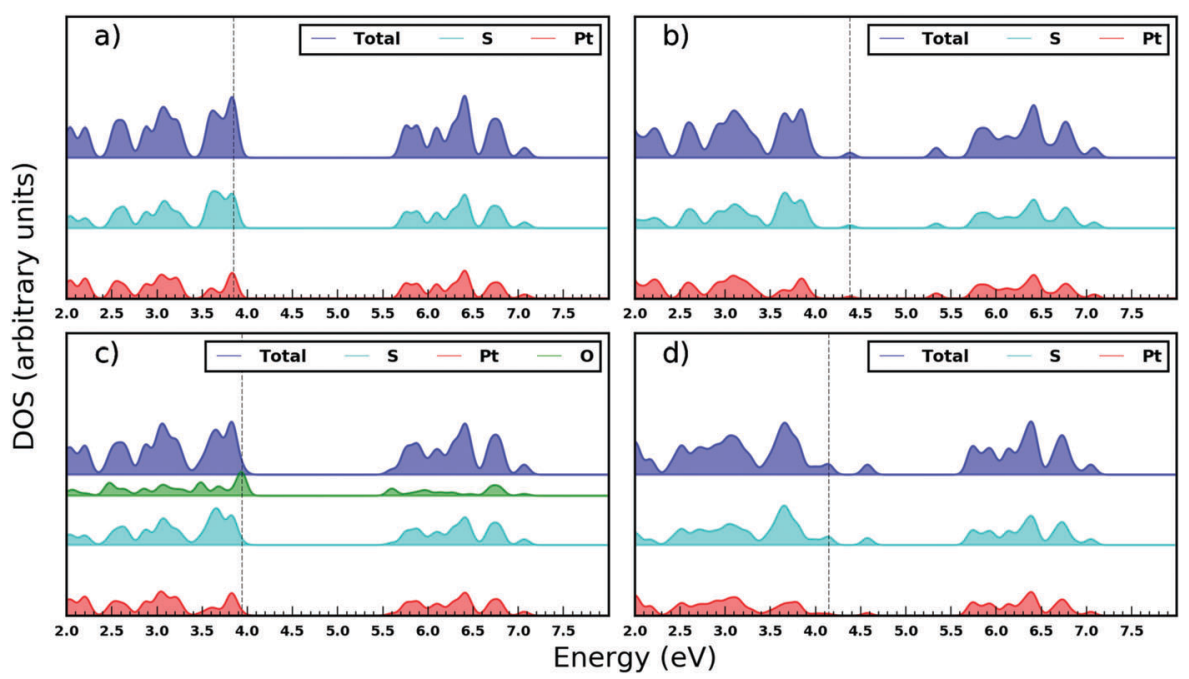

Fig. 13 (a) Shows the DOS for pristine $\mathrm{PtS}_{2}-1 T$ with individual contributions. (b) Shows the DOS for PtS $2-1 T$ with one sulfur vacancy. (c) Shows the DOS with the sulfur vacancy replaced by an oxygen atom. The contribution of oxygen states is relatively higher close to the valence band edge. (d) Shows the impact of a Pt vacancy on the DOS. The vertical dashed line indicates the HOMO level.

any deep gap defects, showing a signature corresponding to a donor state. Most of the impact of the vacancy lies as shallow levels. This is in line with the result reported in ref. 39, where the orbital overlap method has been used for various phases of 2D materials, to determine the presence of deep and shallow defects. We do see a modulation of the DOS, which is considerably larger as compared to the case of $\mathrm{MoS}_{2}$.

Thus the $\mathrm{HfS}_{2}-1 \mathrm{~T}$ phase, unlike $\mathrm{MoS}_{2}$, is a defect tolerant material. The presence of the Hf vacancy in the $\mathrm{HfS}_{2}-1 \mathrm{~T}$ phase leads to a shrinkage in the bandgap, while oxidation of a single chalcogen defect brings back the defective material to its pristine state.

In the specific case of the $\mathrm{PtS}_{2}-1 \mathrm{~T}$ phase, Fig. 13, the presence of a sulfur defect leads to the formation of two defect states close to the CB and VB edges, showing an amphoteric nature. The metal vacancy, on the other hand, creates a defect state close to the valence band, similar to other TMDs. The oxidation of the sulfur vacancy removes the defect state from the gap, as pointed out for the other materials.

The defective selenides and tellurides of $\mathrm{MoX}_{2}$ have a similar behavior to the one reported for $\mathrm{MoS}_{2}$ (the interested reader is invited to consult the DOS corresponding to selenides and tellurides in the ESI $\dagger$ ). Table 1 summarizes the position of the defect states in the different $2 \mathrm{D}$ materials studied. The corresponding DOS is provided in the ESI. $\dagger$ The second column lists the various defect states that are identifiable in the gap or close to edges. $\mathrm{A}-$ or + sign under the defect column shows a state which is close to the CB or $\mathrm{VB}$, respectively, for both metal and chalcogen vacancies. The value shows the corresponding offset from the $\mathrm{CB}$ or VB edges. Additionally, from a device point of view, a $2 \mathrm{D}$ channel should present a sufficiently large bandgap, while a semi-metallic $\left(\sim 0.1 \mathrm{eV} E_{\mathrm{g}}\right) 2 \mathrm{D}$ material may become metallic in the presence of defects.

\subsection{Healing vacancies through reducing agents}

The reactivity of defective $2 \mathrm{D}$ materials with ambient species can lead to a material with degraded electronic properties.
Table 1 Defect states present in different 2D materials w.r.t. the Fermi level. The defect levels are given w.r.t. the conduction and valence band edges of the pristine material (part a in DOS plots). Only additional states in the gap which are clearly identified have been shown. The use of one $K$-point doesn't allow a good resolution for identifying very small changes and edge shifts. A minus sign shows a defect state close to the $C B$ and $a+$ sign shows a defect state close to the VB for each type of defect ( $n$ or $p$ ). A single - or + represents a clear modulation of the CB or VB where a clear state could not be identified or where there is no state in the gap. ${ }^{*} M C$ stands for monochalcogenide

\begin{tabular}{|c|c|c|c|c|c|}
\hline \multirow[b]{2}{*}{$2 \mathrm{D}$} & \multirow[b]{2}{*}{ Phase } & \multicolumn{2}{|c|}{ Chalcogen defect $(\mathrm{eV})$} & \multicolumn{2}{|c|}{ Metal defect (eV) } \\
\hline & & $\Delta \mathrm{CB}$ & $\Delta \mathrm{VB}$ & $\Delta \mathrm{CB}$ & $\Delta \mathrm{VB}$ \\
\hline GaS & $\mathrm{MC}^{*}$ & -0.55 & +0.4 & $-0.2,-0.6$ & \\
\hline GaSe & $\mathrm{MC}^{*}$ & -0.2 & +0.25 & -0.55 & +0.2 \\
\hline $\mathrm{HfS}_{2}$ & $1 \mathrm{~T}$ & - & + & - & + \\
\hline $\mathrm{HfSe}_{2}$ & $1 \mathrm{~T}$ & - & + & - & + \\
\hline $\mathrm{HfSe}_{2}$ & $2 \mathrm{H}$ & -0.2 & & Metallic & Metallic \\
\hline $\mathrm{HfTe}_{2}$ & $2 \mathrm{H}$ & Metallic & & & + \\
\hline InS & $\mathrm{MC}$ & - & +0.35 & -0.1 & +0.25 \\
\hline InSe & MC & - & +0.3 & & +0.2 \\
\hline $\mathrm{MoS}_{2}$ & $2 \mathrm{H}$ & -0.77 & +0.04 & & $+0.2,+0.54,+0.9$ \\
\hline $\mathrm{MoSe}_{2}$ & $2 \mathrm{H}$ & -0.6 & & & $+0.55,+0.75$ \\
\hline $\mathrm{MoTe}_{2}$ & $2 \mathrm{H}$ & -0.45 & & -0.6 & $+0.35,+0.55$ \\
\hline $\mathrm{NiS}_{2}$ & $1 \mathrm{~T}$ & - & + & - & + \\
\hline $\mathrm{PtS}_{2}$ & $1 \mathrm{~T}$ & -0.5 & +0.55 & & $+0.3,+0.75$ \\
\hline $\mathrm{PtSe}_{2}$ & $1 \mathrm{~T}$ & -0.3 & +0.55 & & $+0.35,+0.8$ \\
\hline $\mathrm{PtTe}_{2}$ & $1 \mathrm{~T}$ & -0.3 & & Metallic & Metallic \\
\hline SnS & & & 0.55 & -0.3 & \\
\hline $\mathrm{SnS}_{2}$ & & -0.65 & & & +0.3 \\
\hline $\mathrm{TiS}_{2}$ & $2 \mathrm{H}$ & -0.15 & & NA & NA \\
\hline $\mathrm{TiSe}_{2}$ & $2 \mathrm{H}$ & -0.15 & & & +0.2 \\
\hline $\mathrm{WS}_{2}$ & $2 \mathrm{H}$ & -0.8 & & & $+0.1,+0.55,+1.0$ \\
\hline $\mathrm{WSe}_{2}$ & $2 \mathrm{H}$ & -0.55 & & & $+0.45,+0.7,+0.9$ \\
\hline $\mathrm{WTe}_{2}$ & $2 \mathrm{H}$ & -0.3 & +0.1 & & $+0.35,+0.7$ \\
\hline $\mathrm{ZrS}_{2}$ & $1 \mathrm{~T}$ & - & + & - & + \\
\hline $\mathrm{ZrS}_{2}$ & $2 \mathrm{H}$ & - & & - & \\
\hline $\mathrm{ZrSe}_{2}$ & $1 \mathrm{~T}$ & - & + & - & + \\
\hline $\mathrm{ZrSe}_{2}$ & $2 \mathrm{H}$ & -0.2 & & - & + \\
\hline $\mathrm{ZrTe}_{2}$ & $2 \mathrm{H}$ & - & + & - & + \\
\hline
\end{tabular}

As shown in the earlier section, the presence of defects can lead to gap states which can impact material properties in an 


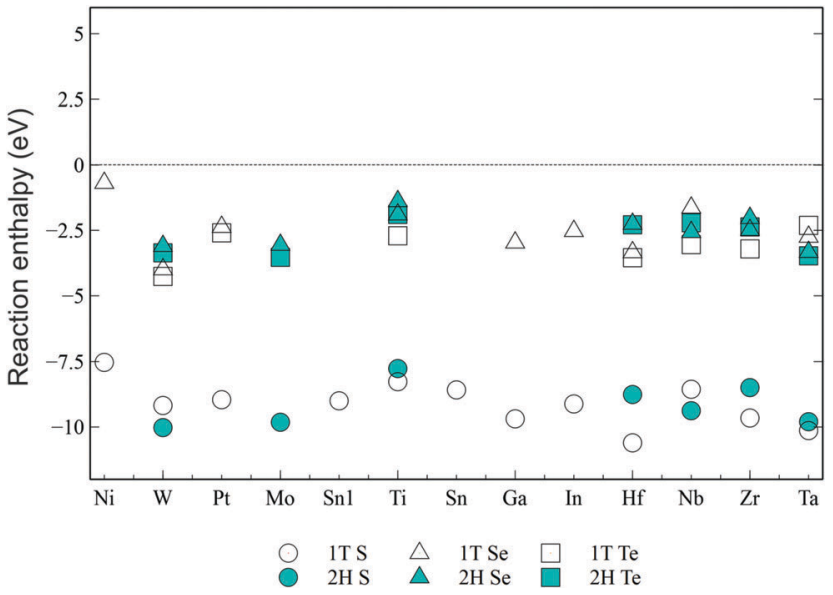

Fig. 14 The enthalpies of healing a single chalcogen vacancy for various 2D materials in the presence of a strong reducing agent $\mathrm{H}_{2} \mathrm{X}(\mathrm{X}=\mathrm{S}, \mathrm{Se}, \mathrm{Te})$ Open symbols and closed symbols correspond to $1 \mathrm{~T}$ and $2 \mathrm{H}$ phases respectively.

undesirable manner. While most of the sulfur vacancies typically introduce defects closer to the CB edge, metal vacancies introduce multiple states spanning a wide range below the midgap for most materials. Additionally, the presence of oxygen may lead to bandgap modulation in $\mathrm{MX}_{2}$ and oxidation of $2 \mathrm{D}$ materials may also lead to a modulation of the $I-V$ characteristics.
Fig. S30 (ESI $\dagger$ ) shows a modulation of the bottom of the conduction band and the top of the valence band with varying oxygen content (O replacing $\mathrm{S}$ atoms in $\mathrm{MoS}_{2}$ ).

Defects and oxidation of defects can lead to a modification of the electronic properties of the 2D material. To tackle this problem, we explore the possibility of healing vacancies using reducing agents $\left(\mathrm{H}_{2} \mathrm{~S}, \mathrm{H}_{2} \mathrm{Te}\right.$ and $\left.\mathrm{H}_{2} \mathrm{Se}\right)$ (Fig. 14). For example, $\mathrm{MoS}_{2}-2 \mathrm{H}$ and $\mathrm{MoS}_{2}-1 \mathrm{~T}$ phases are shown on the same vertical line in Fig. 14. Positive enthalpies show that once the defects are created, it is not possible to heal them in a certain material with a reducing agent. Interestingly, our results suggest that $\mathrm{H}_{2} \mathrm{~S}, \mathrm{H}_{2} \mathrm{Se}$ or $\mathrm{H}_{2} \mathrm{Te}$ have a strong thermodynamic driving force to reduce the defective and oxidized vacancies, restoring the defective $2 \mathrm{D}$ materials to their pristine state. The reaction is more favorable with sulfides than with selenides or tellurides, reflecting the stability of the corresponding pristine 2D sulfides. Selenides and tellurides have a similar drive for being healed.

We observe a similar trend in reduction to that for chalcogen vacancies. The oxidized sulfur vacancies have a higher drive for reduction as compared to their selenide and telluride equivalents. The reduction enthalpies are decreased, as compared to the ones in Fig. 15, showing that it is more difficult to heal an oxidized vacancy. Nonetheless, the difference is not too large and it should thus be possible to heal vacancies using a strong reducing agent, even if the vacancies are oxidized. Note that

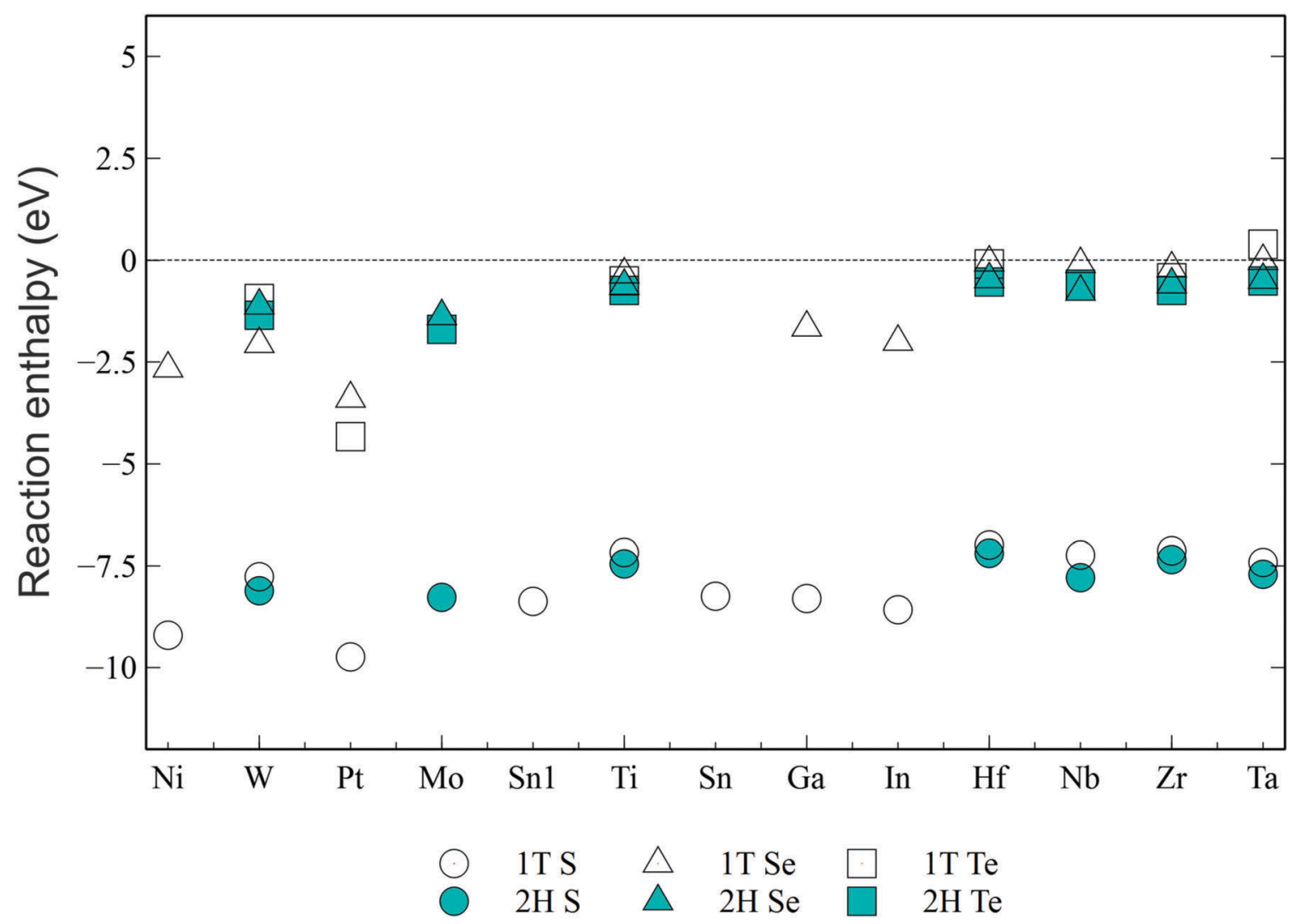

Fig. 15 The enthalpies of healing an oxidized vacancy for various $2 \mathrm{D}$ materials in the presence of a strong reducing agent $\mathrm{H}_{2} \mathrm{X}(\mathrm{X}=\mathrm{S}, \mathrm{Se}, \mathrm{Te})$. Open symbols and closed symbols correspond to $1 \mathrm{~T}$ and $2 \mathrm{H}$ phases respectively. 
while it might be possible to heal defects and counter defect doping using a reducing agent, it might not be possible to fully heal the material. The defect doping also depends on multiple other factors like grain boundaries, reactive $2 \mathrm{D}$ edges, adhesive leftovers from processing, substrate interaction and restricted access to defects for a reducing agent in a multilayer 2D material.

\section{Conclusions}

We studied the thermodynamic aspect of about fifty 2D materials using density functional theory, in the light of the thermodynamic aspects of the defect formation and oxidation processes. The impact of defects on the electronic properties of these 2D materials was studied and the possibility to heal the defects with oxygen-related species or reducing agents (like $\mathrm{H}_{2} \mathrm{~S}$ or $\mathrm{H}_{2} \mathrm{Se}$ ) was also investigated. We found that chalcogen vacancies are the most likely source of defects to be formed, with a relatively low formation enthalpy, independent of the 2D material considered. Formation enthalpies of metal vacancies are in general more endo-energetic and spread over a wide energy distribution window $(\sim 0-20 \mathrm{eV})$. As a result, they are less likely to be formed, with an exception in the case of $\mathrm{SnS}$, where metal vacancies form as easily as the chalcogen vacancies. We also found that pristine $2 \mathrm{D}$ materials are more stable as compared to defective 2D materials, when considering their interaction with the environment. Defective $2 \mathrm{D}$ materials react with different oxidants to varying degrees depending on the ambient species and the material. The interaction energies of $\mathrm{O}_{2}$ with $2 \mathrm{D}$ materials hints towards the fact that the cation present in the $2 \mathrm{D}$ material dictates the strength of the interaction. We also showed that, while defective $2 \mathrm{D}$ materials react with ambient species, the drive towards reaction with high- $\kappa$ surface groups $(-\mathrm{OH})$ is not strong. $\mathrm{Al}_{2} \mathrm{O}_{3}$ and $\mathrm{HfO}_{2}$ surface groups $(-\mathrm{OH})$ have a smaller driving force to react with $2 \mathrm{D}$ materials, either defective or pristine, as compared to $\mathrm{SiO}_{2}$, and bulk high- $\kappa \mathrm{S}$ have a very small driving force to react even with defective $2 \mathrm{D}$ materials. The above analysis shows that $2 \mathrm{D}$ materials are susceptible to undergo formation of defects that can be oxidized under different ambient conditions, being either present during the growth of a dielectric $\left(\mathrm{O}_{2}, \mathrm{H}_{2} \mathrm{O}, \mathrm{O}^{*}, \ldots\right)$ or during the transfer process. The analysis of the electronic properties of pristine and defective $2 \mathrm{D}$ materials sheds more light on the impact of defects from the device perspective. In general, we found that sulfur vacancies create shallow or deep defect states close to the conduction band. These defects are associated with a low enthalpy of formation, which hints towards a natural tendency of 2D materials to adopt n-type behavior due to their chalcogen vacancies. Metal vacancies typically introduce states close to the valence band in general and are associated with a higher formation enthalpy. In general, there is a natural tendency in 2D materials to have defect based doping. Finally, we also found that while the presence of vacancies is likely in various $2 \mathrm{D}$ materials, they can be healed by treatment with a strong reducing agent. Both oxidized and pristine vacancies can potentially be healed using this approach.

\section{Conflicts of interest}

There are no conflicts to declare.

\section{References}

1 N. Z. Haron and S. Hamdioui, Why is CMOS scaling coming to an END? Proceedings - 2008 3rd International Design and Test Workshop, IDT 2008, 2008, 98-103.

2 A. M. Ionescu and $\mathrm{H}$. Riel, Tunnel field-effect transistors as energy-efficient electronic switches, Nature, 2011, 479(7373), 329-337.

3 L. M. Peng, Z. Zhang and S. Wang, Carbon nanotube electronics: Recent advances, Mater. Today, 2014, 17(9), 433-442.

4 D. Bhattacharya and N. K. Jha, FinFETs: From Devices to Architectures, Adv. Electron., 2014, 2014, 1-21.

5 B. Behin-Aein, D. Datta, S. Salahuddin and S. Datta, Proposal for an all-spin logic device with built-in memory, Nat. Nanotechnol., 2010, 5(4), 266-270.

6 F. Schwierz, J. Pezoldt and R. Granzner, Two-dimensional materials and their prospects in transistor electronics, Nanoscale, 2015, 7(18), 8261-8283.

7 G. R. Bhimanapati, Z. Lin, V. Meunier, Y. Jung, J. Cha, S. Das, D. Xiao, Y. Son, M. S. Strano, V. R. Cooper, L. Liang, S. G. Louie, E. Ringe, W. Zhou, S. S. Kim, R. R. Naik, B. G. Sumpter, H. Terrones, F. Xia, Y. Wang, J. Zhu, D. Akinwande, N. Alem, J. A. Schuller, R. E. Schaak, M. Terrones and J. A. Robinson, Recent Advances in TwoDimensional Materials beyond Graphene, ACS Nano, 2015, 9(12), 11509-11539.

8 M. Chhowalla, D. Jena and H. Zhang, Two-dimensional semiconductors for transistors, Nat. Rev. Mater., 2016, 1(11), 16052.

9 L. Banszerus, M. Schmitz, S. Engels, J. Dauber, M. Oellers, F. Haupt, K. Watanabe, T. Taniguchi, B. Beschoten and C. Stampfer, Ultrahigh-mobility graphene devices from chemical vapor deposition on reusable copper, Sci. Adv., 2015, 1(6), e1500222.

10 M. D. Segall, P. J. D. Lindan, M. J. Probert, C. J. Pickard, P. J. Hasnip, S. J. Clark and M. C. Payne, First-principles simulation: ideas, illustrations and the CASTEP code, J. Phys.: Condens. Matter, 2002, 14(11), 2717-2744.

11 A. Gupta, T. Sakthivel and S. Seal, Recent development in 2D materials beyond graphene, Prog. Mater. Sci., 2015, 73, 44-126.

12 H. Van Ngoc, Y. Qian, S. K. Han and D. J. Kang, "PMMAetching-free transfer of wafer-scale chemical vapor deposition two-dimensional atomic crystal by a water soluble polyvinyl alcohol polymer method, Sci. Rep., 2016, 6, 33096.

13 K. Verguts, K. Schouteden, C. H. Wu, L. Peters, N. Vrancken, X. Wu, Z. Li, M. Erkens, C. Porret, C. Huyghebaert, C. Van Haesendonck, S. De Gendt and S. Brems, Controlling Water Intercalation Is Key to a Direct Graphene Transfer, ACS Appl. Mater. Interfaces, 2017, 9(42), 37484-37492. 
14 Y. Guo, D. Liu and J. Robertson, Chalcogen vacancies in monolayer transition metal dichalcogenides and Fermi level pinning at contacts, Appl. Phys. Lett., 2015, 106(17), 173106.

15 J. Hutter, M. Iannuzzi, F. Schiffmann and J. Vandevondele, Cp2k: Atomistic simulations of condensed matter systems, Wiley Interdiscip. Rev.: Comput. Mol. Sci., 2014, 4(1), 15-25.

16 J. Perdew, K. Burke and M. Ernzerhof, Generalized Gradient Approximation Made Simple, Phys. Rev. Lett., 1996, 77(18), 3865-3868.

17 S. Goedecker, M. Teter and J. Hutter, Separable dual-space Gaussian pseudopotentials, Phys. Rev. B: Condens. Matter Mater. Phys., 1996, 54(3), 1703-1710.

18 P. Miró, M. Audiffred and T. Heine, An atlas of twodimensional materials, Chem. Soc. Rev., 2014, 6537-6554.

19 S. Grimme, J. Antony, S. Ehrlich and H. Krieg, A consistent and accurate ab initio parametrization of density functional dispersion correction (DFT-D) for the 94 elements H-Pu, J. Chem. Phys., 2010, 132(15), 154104.

20 H.-S. Ra, D.-H. Kwak and J.-S. Lee, A hybrid $\mathrm{MoS}_{2}$ nanosheetCdSe nanocrystal phototransistor with a fast photoresponse, Nanoscale, 2016, 8(39), 17223-17230.

21 S. Haldar, H. Vovusha, M. K. Yadav, O. Eriksson and B. Sanyal, Systematic study of structural, electronic, and optical properties of atomic-scale defects in the twodimensional transition metal dichalcogenides MX2, Phys. Rev. B: Condens. Matter Mater. Phys., 2015, 92(23), 235408.

22 J. Hong, Z. Hu, M. Probert, K. Li, D. Lv, X. Yang, L. Gu, N. Mao, Q. Feng, L. Xie, J. Zhang, D. Wu, Z. Zhang, C. Jin, W. Ji, X. Zhang, J. Yuan and Z. Zhang, Exploring atomic defects in molybdenum disulphide monolayers, Nat. Commun., 2015, 6, 6293.

23 B. Akdim, R. Pachter and S. Mou, Theoretical analysis of the combined effects of sulfur vacancies and analyte adsorption on the electronic properties of single-layer $\mathrm{MoS}_{2}$, Nanotechnology, 2016, 27(18), 185701.

24 S. Horzum, D. Çakır, J. Suh, S. Tongay, Y.-S. Huang, C.-H. Ho, J. Wu, H. Sahin and F. M. Peeters, Formation and stability of point defects in monolayer rhenium disulfide, Phys. Rev. B: Condens. Matter Mater. Phys., 2014, 89(15), 155433.

25 S.-C. Lu and J.-P. Leburton, Electronic structures of defects and magnetic impurities in $\mathrm{MoS}_{2}$ monolayers, Nanoscale Res. Lett., 2014, 9(1), 676.

26 D. W. Ma, Q. G. Wang, T. X. Li, C. Z. He, B. Y. Ma, Y. A. Tang, Z. S. Lu and Z. X. Yang, Repairing sulfur vacancies in the $\mathrm{MoS}_{2}$ monolayer by using $\mathrm{CO}$, NO and $\mathrm{NO}_{2}$ molecules, J. Mater. Chem. C, 2016, 4(29), 7093-7101.
27 Y. Xu, Y. Li, X. Chen, C. Zhang, R. Zhang and P. Lu, First-principle study of hydrogenation on monolayer $\mathrm{MoS}_{2}$, AIP Adv., 2016, 6(7), 075001.

28 K. Iordanidou, M. Houssa, G. Pourtois, V. V. Afanas'ev and A. Stesmans, Impact of Point Defects and Oxidation on the Electronic Properties of $\mathrm{HfS}_{2}$ Monolayers, ECS J. Solid State Sci. Technol., 2016, 5(11), Q3054-Q3059.

29 H. Chen, Y. Li, L. Huang and J. Li, Intrinsic defects in gallium sulfide monolayer: a first-principles study, $R S C$ $A d v .$, 2015, 5(63), 50883-50889.

30 L. Ao, A. Pham, H. Y. Xiao, X. T. Zu and S. Li, Engineering the electronic and magnetic properties of d0 2D dichalcogenide materials through vacancy doping and lattice strains, Phys. Chem. Chem. Phys., 2016, 18(10), 7163-7168.

31 H.-P. Komsa, J. Kotakoski, S. Kurasch, O. Lehtinen, U. Kaiser and A. V. Krasheninnikov, Two-dimensional transition metal dichalcogenides under electron irradiation: Defect production and doping, Phys. Rev. Lett., 2012, 109(3), 035503.

32 S. Kc, R. C. Longo, R. M. Wallace and K. Cho, Surface oxidation energetics and kinetics on $\mathrm{MoS}_{2}$ monolayer, J. Appl. Phys., 2015, $117(13), 135301$.

33 C. S. Cucinotta, K. Dolui, H. Pettersson, Q. M. Ramasse, E. Long, S. E. O'Brian, V. Nicolosi and S. Sanvito, Electronic Properties and Chemical Reactivity of $\mathrm{TiS}_{2}$ Nanoflakes, J. Phys. Chem. C, 2015, 119(27), 15707-15715.

34 A. Valsaraj, J. Chang, A. Rai, L. F. Register and S. K. Banerjee, Theoretical and experimental investigation of vacancy-based doping of monolayer $\mathrm{MoS}_{2}$ on oxide, $2 \mathrm{D}$ Mater., 2015, 2(4), 045009.

35 D. M. Sim, M. Kim, S. Yim, M. J. Choi, J. Choi, S. Yoo and Y. S. Jung, Controlled Doping of Vacancy-Containing Few-Layer $\mathrm{MoS}_{2}$ via Highly Stable Thiol-Based Molecular Chemisorption, ACS Nano, 2015, 9(12), 12115-12123.

36 C. G. Van De Walle, Universal alignment of hydrogen levels in semiconductors and insulators, Phys. B, 2006, 376-377(1), 1-6.

37 S. Kc, R. C. Longo, R. Addou, R. M. Wallace and K. Cho, Impact of intrinsic atomic defects on the electronic structure of $\mathrm{MoS}_{2}$ monolayers, Nanotechnology, 2014, 25(37), 375703.

$38 \mathrm{H}$. Liu, N. Han and J. Zhao, Atomistic insight into the oxidation of monolayer transition metal dichalcogenides: from structures to electronic properties, RSC Adv., 2015, 5(23), 17572-17581.

39 M. Pandey, F. A. Rasmussen, K. Kuhar, T. Olsen, K. W. Jacobsen and K. S. Thygesen, Defect-Tolerant Monolayer Transition Metal Dichalcogenides, Nano Lett., 2016, 16(4), 2234-2239. 